\title{
Metformin Induced Lactic Acidosis What Is the Effective Treatment?
}

\author{
Manal Alotaibi1 ${ }^{*}$, Farhan Ali1, Sharon Leung², Rebecca Kent ${ }^{2}$ \\ ${ }^{1}$ University of Maryland Medical Center, Baltimore, MD, USA \\ ${ }^{2}$ Montefiore Medical Center, Bronx, NY, USA \\ Email: malotaibi@som.umaryland.edu
}

How to cite this paper: Alotaibi, M., Ali, F., Leung, S. and Kent, R. (2020) Metformin Induced Lactic Acidosis What Is the Effective Treatment? Open Journal of Nephrology, 10, 11-17.

https://doi.org/10.4236/ojneph.2020.101002

Received: December 26, 2019

Accepted: January 28, 2020

Published: January 31, 2020

Copyright $\odot 2020$ by author(s) and Scientific Research Publishing Inc. This work is licensed under the Creative Commons Attribution International License (CC BY 4.0).

http://creativecommons.org/licenses/by/4.0/

\section{(c) (i) Open Access}

\begin{abstract}
Metformin is known to be an effective treatment for patients with type 2 diabetes mellitus. Metformin induced lactic acidosis (MALA) is a serious illness which should identify and treat urgently. In this review article, we will present our own data and review the literature to highlight the importance of recognizing MALA; causes, presentations and the proper treatment, even though there are few published studies discussing MALA treatment modalities. The two cases that we presented here were patients admitted to the ICU and undergoing hemodynamic instability, which improved after removal of Metformin and clearance of associated lactic acidosis with SLED and CVVH. A case could be made for SLED and CVVH as modalities of choice for patients with MALA and hemodynamic instability, but further research is needed in this direction.
\end{abstract}

\section{Keywords}

MALA (Metformin Induced Lactic Acidosis), AKI (Acute Kidney Injury), CRRT (Continuous Renal Replacement Therapy)

\section{Introduction}

Metformin is one of the first-line treatments for patients with type 2 diabetes mellitus [1]. Metformin reduces the risk of mortality and morbidity in patients with diabetes. It considers a safe drug, with some limitations. Phenformin, an earlier biguanide was withdrawn from the market after associations with lactic acidosis. Lactic acidosis is one of rare but life-threatening metformin side effects especially in the presence of acute kidney injury (AKI) [1]. Metformin induced lactic acidosis (MALA) is a serious illness which should identify and treat ur${ }^{*}$ First author and author of reference. 
gently. In this review article, we will present our own data and review the literature to highlight the importance of recognizing MALA; causes, presentations and the proper treatment, even though there are few published studies discussing MALA treatment modalities.

\section{Mechanism of Action}

Metformin reduces glucose levels by increasing insulin sensitivity, reducing hepatic glucose release and increasing muscle uptake [1] [2]. It prevents hepatic gluconeogenesis by reducing the lactate reuptake. That leads to increases in plasma lactate levels. When metformin concentrations increased in the plasma as occur in patients with AKI or when lactate production or clearance disturbed, MALA occurs [1] [2].

\section{Incidence and Mortality Rate}

Metformin prescriptions have been progressively increasing over time with increasing the reported cases of MALA. MALA has become not so uncommon in the ICU setting. The incidence rate of MALA is estimated at 2 - 9 cases per 100,000 persons receiving metformin yearly. Approximately $1 \%$ of total patients admitted to intensive care units (ICU) due to MALA. A retrospective study over the period of six years in Piedmont and Aosta Valley regions was done on 5000 patients who admitted to the ICU and received hemodialysis reported one hundred and seventeen cases of AKI caused by MALA [1].

The mortality rate of MALA is high up to $30 \%-50 \%$ yearly [2] [3]. However, there is no large study has examined the incidence, mortality rate, clinical pictures and outcome of AKI in MALA.

\section{Presentations}

MALA might present with non-specific symptoms like gastroenterology symptoms, fatigue, confusion or altered mental status (AMS). The risk will increase in patient with acute kidney injury, chronic kidney injuries, or heart failure who are diabetic on metformin. It is important to consider it as one of the differential diagnosis when treating unexplainable metabolic acidosis with high anion gab and lactic acidosis [4] [5]. MALA should be suspected in patients presenting with hemodynamic instability, respiratory failure, profound hypotension or shock with laboratory abnormality of wide anion gap metabolic acidosis and high lactate level, even when they are non-diabetic [6].

There are few case reports where patients attempted suicide by using metfor$\mathrm{min}$ and they do not have diabetes. There is a reported case of 44 years old Japanese woman was attempted suicide by ingesting 10 tablets of metformin $500 \mathrm{mg}$ and drinking approximately $600 \mathrm{~mL}$ of alcohol. She was presented with altered mental status and shock, Continuous intravenous administration of noradrenalin $(0.13 \mu \mathrm{g} / \mathrm{kg}$ per minute) was started. Laboratory findings included a lactate level of $119 \mathrm{mg} / \mathrm{dL}(13.2 \mathrm{mmol} / \mathrm{L})$, bicarbonate of $14.5 \mathrm{mmol} / \mathrm{L}$, and serum met- 
formin concentration of $1138 \mathrm{ng} / \mathrm{mL}$. She was diagnosed as having MALA worsened by alcohol. High-flow high-volume intermittent hemodiafiltration was started for 6 hours. Consequently, serum metformin concentration decreased to $136 \mathrm{ng} / \mathrm{mL}$ and noradrenalin administration became unnecessary to maintain normal vital signs. On hospital day 12 , she was moved out from the ICU to the psychiatry ward [7]. Another five patients admitted to Chang Gung Memorial Hospital with severe lactic acidosis and diagnosed as MALA. Their renal functions were normal before this onset. Two of them had attempted suicide by taking large amounts of metformin. One of them has End Stage Renal Diseases (ESRD) on regular hemodialysis. One of the patients who had taken metformin to attempt suicide was not diabetic [8].

\section{Treatment}

MALA management is controversial and mostly supportive. It may include activated charcoal, bicarbonate infusion, hemodialysis, or continuous venovenous hemofiltration [9] [10]. Intermittent hemodialysis and continuous renal replacement therapy (CRRT) are reported as effective MALA treatment, although it is poorly documented with a few case series and reports available worldwide.

Metformin is mostly absorbed in the upper small intestine. Unabsorbed drug accumulates in the mucosa of the bowel and is execrated in the feces. Metformin is a relatively small molecule $(16 \mathrm{kDa})$ that is non-protein bound and has a $50 \%$ $60 \%$ oral bioavailability. It circulates in the plasma unbound and is eliminated by the kidneys. Because of these properties, metformin can be removed from the blood by renal replacement therapy. Besides, metformin has a large volume of distribution $(63 \mathrm{t} 276 \mathrm{~L})$. This might make continuous replacement more effective than intermittent hemodialysis [2] [3] [11].

The choice of the specific modality and treatment time of renal replacement therapy (RRT) to treat MALA and achieve stability is still debated with lack of large randomized or large control published studies.

Greco and his colleagues published a study of ten patients who admitted to the ICU with acute kidney injury and hemodynamic instability for suspected MALA. Patients underwent 16 hours of Sustained Low Efficiency Dialysis (SLED) sessions performed with either conventional dialysis machines or machines for continuous RRT (CRRT). A 2-compartment open-infusion pharmacokinetic model with first-order elimination was fitted to each subject's serum concentration-time data to model post-SLED rebound and predict the need for further treatments. Two patients died within $24 \mathrm{~h}$ after SLED start. Three patients needed one further dialysis session. Surviving patients were dialysis-free at discharge. Metformin levels were in the toxic range at baseline and decreased rapidly by the end of SLED without differences according to the dialysis machine [12].

The outcomes of systemic evaluation per Peter in patients with MALA admitted to the intensive care unit showed the mortality rate of patients who received 
dialysis was the same to that of patients who were not dialyzed. However, it was the more acutely ill patients who actually received dialysis. This suggests that dialysis was beneficial in preventing a higher mortality rate in those who required dialysis [9].

We presented two cases in which hemodynamic stability returned within 12 hours after the initiation of renal replacement therapy in patients with MALA induced by acute kidney injury.

\section{Case 1}

A 58-year-old man with a history of diabetes mellitus (DM) was admitted for generalized weakness, fatigue, nausea, and vomiting for four days.

On admission, his vitals were stable except he was tachypneic to 48 breaths/min. He was oliguric.

Initial laboratory results showed Sodium 136, Potassium 7.5, Chloride 92, Bicarbonate 9, BUN 92, Creatinine 14.2, from a baseline of 1.3, PH 7.00 and lactate 17.

The patient's medications included Actos, Benazepril, Glipizide, and Metfor$\min$.

One hour upon arrival at the ICU, the patient developed profound hypotension and hypothermia (92F) which required four vasopressors to maintain a mean arterial pressure of 6.5. PH was unmeasurable (i.e., less than 6.85), lactate 22; serum potassium 8.0 and bicarbonate less than 6 . Sustained Low-Efficiency Dialysis (SLED) was initiated because of the profound acidosis and hemodynamic instability. The patient's hemodynamics dramatically improved within the first 12 hours. No clinical or microbiological infections were identified. The metformin level prior to SLED was $9.9 \mathrm{mcg} / \mathrm{mL}$ and was $6.1 \mathrm{mcg} / \mathrm{mL} 10$ hours afterward (normal range: $1-2 \mathrm{mcg} / \mathrm{mL}$ ). The patient recovered uneventfully and was discharged on day 6 .

\section{Case 2}

A 78-year-old woman with a history of diabetes mellitus and osteoporosis was found unresponsive at home.

Initial blood pressure was 40/20; pulse was 26 . Temperatures were unmeasurable, i.e., below $84 \mathrm{~F}$.

Outpatient medications include Metformin and Furosemide. Five milligrams of Zoledronic acid was administrated intravenously 19 days prior to admission for osteoporosis.

Initial laboratory results showed Sodium 139, Potassium 6.5, Chloride 97, Bicarbonate less than 6, BUN 104, Creatinine 5.7, PH 6.68 and lactate 11.1.

Two vasopressors were required. Because of the severe metabolic acidosis, Continuous Venovenous Hemofiltration (CVVH) was initiated. Before the infusion, her creatinine was 1.0. The patient's hemodynamics dramatically improved within the first 12 hours. No organism was identified from all cultures. Serum 
Metformin level on admission was $19 \mathrm{mcg} / \mathrm{mL}$ and one day after initialing CVVH was $3.0 \mathrm{mcg} / \mathrm{mL}$. The patient was transferred to a medicine floor on day 6. Another case was reported for a 71 years old man presented with gradual gastrointestinal symptoms and found to have shock with lactic acidosis. Patient was stabilized within 18 hours after starting urgent CRRT with completely normalized PH [4].

\section{Discussion}

Based on our cases and some published cases; the requirement of vasopressor medications in patients who suffered from severe lactic acidosis secondary to metformin toxicity with extreme hemodynamic instability, dramatically decreased with using of CRRT [13].

CRRT, in addition to treating renal failure and volume correction, allowed a rapid correction of lactic acidosis and recovery from MALA. An excellent prognosis with hemodynamic stability, metabolic acidosis control and elimination of metformin were performed by using CRRT in a three years period retrospective study which was done per Geoffray Keller, etc. Their study included sex patients who admitted to the intensive care unit with severe lactic acidosis secondary to metformin uses. The mean $\mathrm{pH}$ and mean lactate was 6.9260.20 and 14.465.1 $\mathrm{mmol} / \mathrm{l}$, respectively. Early CRRT comprised either venovenous hemofiltration $(n=3)$ or hemodiafiltration $(n=3)$ with a mean effluent flow rate of 3466 $\mathrm{mL} / \mathrm{kg} / \mathrm{h}[4]$.

The mode and the duration of dialysis which is most effective to treat MALA are still unclear.

There is a retrospective study for the period of 10 years which showed intermittent hemodialysis is effective to treat MALA. The study was done in the ICU setting with enrollment of forty-two patients with MALA. 13 patients with intentional overdoses and 29 patients with incidental overdose. It showed that a cumulative hemodialysis duration of 15 hours was associated with the return of metformin level to the normal therapeutic range in $74 \%$ of patients who were in acute renal failure and required HD [5].

Also in a few cases, both intermittent hemodialysis and CRRT were used with great outcomes in patients with MALA.

A published case report in Nephrology Dialysis Transplantation journal showed dramatic improvement in a patient with severe lactic acidosis and extremely high serum metformin concentrations after starting hemodialysis with high-volume continuous veno-venous haemofiltration (CVVH). At first, he started on intermittent hemodialysis for $11 \mathrm{~h}$ (with one $\mathrm{h}$ interruption) without any improvement and with progressive hemodynamic instability. Hemodialysis treatment was stopped, and CVVH with bicarbonate substitute was started. However, due to his instability, a second CVVH machine with femoral catheter access was started. Under this high-volume CVVH (blood flow $300 \mathrm{~mL} / \mathrm{min}$, ultrafiltration $5 \mathrm{l} / \mathrm{h}$ ) for $16 \mathrm{~h}$, lactic acidosis improved, the hemodynamic situation 
of the patient stabilized, and he was discharged from the ICU [8].

Another case report published for two patients with severe lactic acidosis secondary to metformin overdoses who required approximately 25 hours of HD to eventually make a complete recovery [6]. Studies showed that increase in ionized calcium during hemodialysis improves left ventricular contractility. Bicarbonate hemodialysis has been shown to improve hemodynamic stability [2].

Excellent outcomes were also reported with using continuous renal replacement therapy.

A case of a 75-year-old woman with metabolic acidosis as a result of metformin assumption, treated by renal replacement therapy (CRRT) with continuous veno-venous hemodiafiltration (CVVHDF). After a short treatment period there was a reduction in Lactates (from $16.8 \mathrm{mmol} / \mathrm{L}$ to $12.6 \mathrm{mmol} / \mathrm{L}$ ) and a progressive improvement of acidosis. In 72 hours the recovery of diuresis and subsequent suspension of CRRT was achieved [14].

Peritoneal dialysis may also be used to treat MALA with good outcome but more evidence is needed. We reviewed a case report for a 14-year-old African girl who ingested an unknown amount of metformin intentionally after an argument with her mother. She was treated with hemodialysis and peritoneal dialysis with resolving of lactic acidosis [15].

\section{Conclusions}

MALA is a serious and non-uncommon problem which can be seen in the ICU. Thus, a high level of suspicion of MALA in patients with acute kidney injury who are taking metformin is particularly critical in this situation. It also should be suspected in patients presenting with lactic acidosis, even when they are non-diabetic.

Urgent treatments including intermittent hemodialysis vs CRRT are required towards rapid recovery and stabilization. Large series of reports and controlled studies are needed to better determine the duration and most effective dialysis modality in patients with MALA.

\section{Conflicts of Interest}

The authors declare no conflicts of interest regarding the publication of this paper.

\section{References}

[1] Mariano, F., et al. (2017) Metformin-Associated Lactic Acidosis Undergoing Renal Replacement Therapy in Intensive Care Units: A Five-Million Population-Based Study in the North-West of Italy. Blood Purification, 44, 198-205.

[2] Peters, N., et al. (2008) Metformin-Associated Lactic Acidosis in an Intensive Care Unit. Critical Care, 12, R149. https://doi.org/10.1186/cc7137

[3] DeFronzo, R., Fleming, G.A., Chen, K. and Bicsak, T.A. (2016) Metformin-Associated Lactic Acidosis: Current Perspectives on Causes and Risk. Metabolism, 65, 20-29. 
[4] Nakamura, A., et al. (2017) Metformin-Associated Lactic Acidosis Treated with Continuous Renal Replacement Therapy. BMJ Case Reports, 2017, bcr2016218318. https://doi.org/10.1136/bcr-2016-218318

[5] Moioli, A., et al. (2016) Metformin Associated Lactic Acidosis (MALA): Clinical Profiling and Management. Journal of Nephrology, 29, 783-789.

https://doi.org/10.1007/s40620-016-0267-8

[6] Chang, C.T., Chen, Y.C., Fang, J.T. and Huang, C.C. (2002) Metformin-Associated Lactic Acidosis: Case Reports and Literature Review. Journal of Nephrology, 15, 398-402.

[7] Suzuki, K., et al. (2018) Effect of High-Flow High-Volume-Intermittent Hemodiafiltration on Metformin-Associated Lactic Acidosis with Circulatory Failure: A Case Report. Journal of Medical Case Reports, 12, 280. https://doi.org/10.1186/s13256-018-1809-6

[8] Panzer, U., Kluge, S., Kreymann, G. and Wolf, G. (2004) Combination of Intermittent Haemodialysis and High-Volume Continuous Haemofiltration for the Treatment of Severe Metformin-Induced Lactic Acidosis. Nephrology Dialysis Transplantation, 19, 2157-2158. https://doi.org/10.1093/ndt/gfh337

[9] Finkle, S.N. (2009) Should Dialysis Be Offered in All Cases of Metformin-Associated Lactic Acidosis? Critical Care, 13, 110. https://doi.org/10.1186/cc7161

[10] Dichtwald, S., Weinbroum, A.A., Sorkine, P., Ekstein, M.P. and Dahan, E. (2012) Metformin-Associated Lactic Acidosis Following Acute Kidney Injury. Efficacious Treatment with Continuous Renal Replacement Therapy. Diabetic Medicine, 29, 245-250. https://doi.org/10.1111/j.1464-5491.2011.03474.x

[11] Alivanis, P., Suzuki, K., Imai, H. and Katayama, N. (2006) Metformin-Associated Lactic Acidosis Treated with Continuous Renal Replacement Therapy. Clinical Therapeutics, 28, 396-400.

[12] Greco, P., et al. (2019) Sustained Low-Efficiency Dialysis for Metformin-Associated Lactic Acidosis in Patients with Acute Kidney Injury. Journal of Nephrology, 32, 297-306. https://doi.org/10.1007/s40620-018-00562-2

[13] Keller, G., et al. (2011) Management of Metformin-Associated Lactic Acidosis by Continuous Renal Replacement Therapy. PLoS ONE, 6, e23200. https://doi.org/10.1371/journal.pone.0023200

[14] Ferrario, M., Apicella, A., Della Morte, M. and Beretta, E. (2018) A Case of Severe Metformin-Associated Lactic Acidosis Treated with CVVHDF and Regional Anticoagulation with Sodium Citrate. Giornale italiano di nefrologia, 35, 2018-vol5.

[15] Elmezughi, K. and Ekpebegh, C. (2019) Metformin-Associated Lactic Acidosis Treated Successfully by Peritoneal Dialysis in a Resource Limited Setting: Case Report. The Pan African Medical Journal, 32, 112.

https://doi.org/10.11604/pamj.2019.32.112.18271 